\title{
Subclinical Onychomycosis in Apparently Healthy Adults
}

\author{
Alejandra Angulo-Rodríguez ${ }^{\mathrm{a}}$ Heidi Hernández-Ramírez ${ }^{\mathrm{b}}$ \\ María Elisa Vega-Memije ${ }^{b}$ Sonia Toussaint-Caire ${ }^{b}$ Gabriela Moreno-Coutiño ${ }^{a}$ \\ aMycology Department, Hospital General Dr. Manuel Gea González, Mexico City, Mexico; \\ bermatopathology Department, Hospital General Dr. Manuel Gea González, Mexico City, Mexico
}

\section{Keywords}

Subclinical onychomycosis - Apparently healthy nails .

Onychomycosis in healthy subjects

\begin{abstract}
Introduction: Onychomycosis is a frequent chronic nail infection, with a worldwide prevalence of $5.5 \%$ making it the most common nail disease, and its incidence increases with age. Clinically, it causes discoloration and thickening of the nail plate and may be accompanied by onycholysis. However, little is known of the subclinical infection. Methods: We studied adult male and female outpatients auto-referred as healthy and that had healthy appearing toenails. Nail distal clippings were obtained from the right first toenail. This sample was stained with PAS and observed by an expert dermatopathologist searching for fungal structures. Results: A total of 32 samples were included: 8 from men (25\%) and 24 from women (75\%), with ages ranging from 27 to 66 years (mean age of 43 years). Twenty-four patients did not present any histopathological finding suggestive of infection (75\%), while 7 patients had a single finding (spores or hyphae) (21.8\%), and 1 patient had both findings (3.12\%). Discussion/Conclusion: We found 4 patients with yeasts, 3 with hyphae, and 1 patient with a combined infection with both
\end{abstract}

yeasts and hyphae (3.1\%). These add up to $25 \%$ of the clinically apparent healthy nails. Our results show that we still have much to learn from the initial stages of onychomycosis and that our population probably has higher incidence of this nail disease, so we must be alert to subtle nail changes. As our participants signed an informed consent, we will contact those that resulted positive for follow up consultations.

(c) 2021 S. Karger AG, Basel

\section{Introduction}

Onychomycosis is a frequent nail infection, with a worldwide prevalence of $5.5 \%$, making it is the most common nail disease, and its incidence increases with age. Clinically, it causes discoloration and thickening of the nail plate, and may be accompanied by onycholysis [1].

This disease has peculiar characteristics, as it is chronic and usually runs an indolent course, so the exact time of initiation of the clinical changes remains unknown for the most part. It may affect fingernails but is mostly seen on toenails where risk factors play a very important role, such as occlusive footwear, tinea pedis, repetitive trauma, venous insufficiency, and immunosuppression such as diabetes or patients living with HIV/AIDS. Also, a famil- karger@karger.com

www.karger.com/sad

Karger
(C) 2021 S. Karger AG, Basel

(ancel González

Colonia Sección XVI, Tlalpan

Mexico City 14080 (Mexico)

gmorenocoutino@gmail.com 
ial predisposition has been documented [2]. Nearly all authors use the clinical classification that divides onychomycosis in 4 categories: distal and lateral subungual onychomycosis, proximal white onychomycosis, superficial white onychomycosis, and total dystrophic onychomycosis. The first, is the most common presentation, and the last is the end stage of any of the others if given enough time. This classification is useful for the clinical evaluation to determine the required treatment modality, and in subsequent visits, for assessment of treatment response.

The main etiological agents are dermatophytes, and of these, Trichophyton rubrum is the most common, followed by T. mentagrophytes. Also, non-dermatophyte moulds and yeasts can be responsible for the infection.

For diagnosis, the gold standard continues to be $\mathrm{KOH}$ mount and culture, which allows species identification. However, other methods have been introduced that can yield good results for those that lack access to a mycology laboratory. For example, a distal nail clipping processed as a regular PAS stain can highlight the fungal structures intermingled with the keratinocytes displaying good sensitivity and specificity [3].

Some authors have reported subclinical onychomycosis, that is, detection of fungal structures within the nail structure without clinical signs of nail disease. One of the diabetic patients had an incidence of 7.5\% [4].

Another study found subclinical onychomycosis in PAS-stained distal nail clippings in $1.5 \%$ of apparently healthy individuals and $17 \%$ of patients with tinea pedis diagnosis, supporting the fact that tinea pedis is a predisposing factor for onychomycosis [5]. Yet another study, this one in patients living with HIV/AIDS, which is a group known to have a higher rate of onychomycosis of up to $44 \%$ compared to non-immunosuppressed same aged patients, found no evidence of subclinical onychomycosis [6]. So, we decided to examine this time, the nails of auto reported healthy patients, without clinical evidence of onychomycosis.

\section{Materials and Methods}

This is an observational, descriptive, prospective, and transversal study approved by our institutional investigation and Ethics Committee, which included adults attending the outpatient consultation of the Mycology section of Dr. Manuel Gea González General Hospital between August 2018 and March 2020. We included auto-referred as healthy patients, meaning those without chronic comorbidities or regular pharmacological treatments, who had normal appearing nails on clinical examination. Individuals with no history of previous antifungal treatment either top-

Subclinical Onychomycosis in Healthy Adults
Table 1. General findings on histopathology

\begin{tabular}{llc}
\hline Condition & Patients, $n$ & Percentage, \% \\
\hline Healthy & 24 & 75 \\
1 finding & 7 (4 yeasts, 3 hyphae) & 21.8 \\
2 findings & 1 (yeasts + hyphae) & 3.12 \\
\hline Total & 32 & 100 \\
\hline
\end{tabular}

Table 2. Non-fungal findings in the total sample

\begin{tabular}{llc}
\hline Finding & Patients, $n$ & Percentage, \% \\
\hline Bacteria & 19 & 59.37 \\
Granular parakeratosis & 15 & 50 \\
Clefts & 20 & 62.5 \\
Serous deposits & 11 & 34.37 \\
Onychokaryosis & 32 & 100 \\
\hline
\end{tabular}

ical or systemic during the past 12 months, as well as those without clinical signs of any onychopathy were invited to participate. An upskilled dermatologist accomplished toenail examination, with subsequent written informed consent acquisition. Nail clippings from the distal border of the right foot first toenail were obtained and processed for periodic acid-Schiff (PAS) staining on the histopathology laboratory. These samples were observed by 2 trained dermatopathologists in search for fungal elements, although samples with bacteria were also reported.

\section{Results}

A total of 32 samples were included: 8 from men (25\%) and 24 from women (75\%), with ages ranging from 27 to 66 years old (mean age of 43 years).

Twenty-four patients did not present any histopathological finding suggestive of infection (75\%), while 7 patients had a single finding $(21.8 \%)$, and 1 patient had 2 findings $(3.12 \%)$. From the 7 patients with a single finding, 4 had yeasts (12.5\%), and 3 had hyphae $(9.3 \%)$, which in all cases, invaded the nail plate. One patient presented both findings, hyphae and yeasts (3.12\%), all shown in Table 1.

Additionally, bacteria were identified in almost $60 \%$ of the samples, and also, the samples analysis revealed that 16 patients had granular parakeratosis (50\%), 20 patients had clefts $(62.5 \%), 11$ patients had serous deposits $(34.37 \%)$, and all of the patients had onychokaryosis (100\%) Table 2. We did not find differences between men and women in any of the results obtained.

Skin Appendage Disord 2021;7:180-182 181 


\section{Discussion/Conclusion}

We found 4 patients with yeasts, 3 with hyphae and 1 patient with a combined infection of both yeasts and hyphae (3.1\%). These fungal structures were seen invading the nail plate which supports a true infection and not contamination. All our positive fungal findings add up to $25 \%$ of the apparently healthy nails. This is significantly higher of Walling's report of 1.5 and $17 \%$ of healthy nails without and with tinea pedis, respectively, and is also higher to the report of Elbendary who found $7.5 \%$ of subungual onychomycosis in patients living with diabetes.

The immune response against onychomycosis is through the Th1 pathway. The haplotypes HLA DR4 and HLA DR 6 are suspected to have a protective effect against onychomycosis, while haplotypes HLD DR8 and HLA DR1 are believed to confer susceptibility. It has been proposed that individuals with specific mutations affecting pattern recognition receptors or the interleukin-17 and interleukin-22 pathways may be more susceptible to onychomycosis and treatment resistance. So, probably it would be ideal that people with these alterations are detected and treated aggressively prior to disease progression [7].

Parallel to the fungal detection, we also found a high number of bacteria in other ways healthy nails and in subclinical onychomycosis cases, but apparently, this finding is not considered either a predisposing or a risk factor for the development of onychomycosis. Onychokariosis, defined like onychocytes with visible nuclei, is a frequent sign, reported by Trevisan et al. [8] as more frequent in onychomycosis (53\%) compared to normal nails (41\%), although there is no statistically significant difference. It has also been noted as less common in psoriasis (7\%), although its precise relation to the disease is still unknown.

Likewise, clefts and parakeratosis are not contemplated exclusive nor a characteristic finding in fungal nail infections. Our results show that we still have much to learn from the initial stages of onychomycosis and of the meaning of the histopathological findings and that our population probably has higher incidence of this nail disease, so we must be alert to subtle nail changes.

As our participants signed an informed consent, we will contact those who resulted positive for follow up consultations, as the presence of scarce hyphae indicate that they are very probably in an early infection stage. Ideally, they will be evaluated during and after treatment to register their progress.

\section{Statement of Ethics}

This research was approved by the Ethics Committee of the institution with the registration number 06-126-2018, and we state that it was conducted ethically in accordance with the World Medical Association Declaration of Helsinki.

\section{Conflict of Interest Statement}

The authors have no conflicts of interest to declare.

\section{Funding Sources}

No funding was received.

\section{Author Contributions}

Alejandra Angulo-Rodríguez: contributed summoning the participants and taking samples and contributed also with the manuscript writing. Heidi Hernández-Ramírez: contributed with the examination of the nail samples and analyzed the results. María Elisa Vega-Memije: contributed with the examination of the nail samples and analyzed the results. Sonia Toussaint-Caire: contributed to the idea of the work and the manuscript writing. Gabriela Moreno-Coutiño: contributed with the development of the work, coordinated the study, and helped with the manuscript writing.

\section{References}

1 Ghannoum M, Isham N, Catalano V. A second look at efficacy criteria for onychomycosis: clinical and mycological cure. Br J Dermatol. 2014;170(1):182-7.

2 Lipner SR, Scher RK. Onychomycosis. Clinical overview and diagnosis. J Am Acad Dermatol. 2019;80:835-51.

3 Jung MY, Shim JH, Lee JH, Lee JH, Yang JM, Lee DY, et al. Comparison of diagnostic methods for onychomycosis, and proposal of a diagnostic algorithm. Clin Exp Dermatol. 2015;40(5):479-84.
4 Elbendary A, El Tawdy A, Zaki N, Alfishawy $M$, Rateb A. Subclinical onychomycosis in patients with type II diabetes. Dermatol Reports. 2015;7(3):6099.

5 Walling HW. Subclinical onychomycosis is associated with tinea pedis. Br J Dermatol. 2009;161(4):746-9.

6 Contreras-Rivera C, Toussaint-Caire S, Fernández-Martínez R, Moreno-Coutiño G. Subclinical onychomycosis: not present in HIV population? Skin Appendage Disord. 2019;5:155-7.
7 Gupta AK, Carviel J, Shear NH. Onychomycosis and chronic fungal disease: exploiting a commensal disguise to stage a covert invasion. J Cutan Med Surg. 2018 May/Jun;22(3): 318-22.

8 Trevisan F, Werner B, Pinheiro RL. Nail clipping in onychomycosis and comparison with normal nails and ungual psoriasis. An Bras Dermatol. 2019;94(3):344-7. 